\title{
Improving Mobile Device Interaction for Parkinson's Disease Patients via PD-Helper
}

\author{
Farzana Jabeen ${ }^{1,2}, \quad$ Linmi Tao ${ }^{1}, \quad$ Yirou Guo ${ }^{3}, \quad$ Shiyu Zhang ${ }^{4}, \quad$ Shanshan $\mathrm{Mei}^{5}$ \\ ${ }^{1}$ Key Laboratory of Pervasive Computing, Ministry of Education, Beijing China \\ ${ }^{1}$ Dept. of Computer Science \& Technology, Tsinghua University, Beijing China \\ ${ }^{2}$ National University of Science and Technology, Islamabad, Pakistan \\ ${ }^{3}$ Department of Electronic Engineering, Beihang University; International School, BUPT, Beijing, China \\ ${ }^{5}$ Neurology Department, Xuan Wu Hospital, Beijing, China
}

\begin{abstract}
In current era, smart devices play vital role in social connectivity and performing routinely activities. The motor and non-motor (rigidity, tremor at rest, dysarthria, slowness of movement, and depression) symptoms of Parkinson's disease (PD) have definite negative impacts on patients' interaction with smart devices. An adaptable interface named PD-Helper is designed and developed to meet the physical capabilities as well as social needs of patients and to improve the accessibility via smart devices. Single touch interaction is provided via scanning selection technique, video and web user controls and input method editor (IME) application allows PD patients to access web browsing, entertainment like watching videos, typing text and contacting medical stuff. 40 users with PD, participated in the evaluation process of the PD-Helper. Preliminary results are promising and satisfactory. Further studies are in progress to attest the PD helper potential, such as improving the quality of life of people with other Neurological disorders.
\end{abstract}

Keywords-component; Parkinson's Disease, Mobile Interaction, Accessibility, Single touch interaction, interaction with smart devices

\section{INTRODUCTION}

In current society, Parkinson's disease (PD) is a progressive degenerative disorder that affects the nervous system and consequently decreases the quality of life (QOL) effecting more than $1 \%$ of the patients worldwide and in China $[8,13]$. As smart devices becoming an indispensable part of human life, PD also inevitably need to use modern communication products. Smart technology has evolved the ways of interaction such as input becomes based on tiny touch screens, on screen keyboards, on gesture and finger input [1]. But the physiology and psychology of the patients with $\mathrm{PD}$ are very different from the target customers of smart phones [1,8,22]. Cardinal symptoms of PD like rigidity and tremor, neurological disorders like insomnia and severe fatigue, undermined speech and voice capabilities are likely to reduce the individual's mobility and autonomy and thus affect the way they interact with smart devices [8].

At present, the smart device with less functions can solve the basic needs of the special groups, but cannot meet the fashion need of the special groups for health, recreation and social demand [1]. The problems of existing smart devices and computers are divided into three sectors: user interface, interaction design, physical capabilities. As for user interface, decorative fonts prevent patients with visual problems reading text easily [5], patients with visual problems find it hard to adapt

DOI reference number: 10.18293/SEKE2019-153 to the brightness changes between screens $[1,4]$, elder patients may have problems with perception of the application and the devices itself and verbiage in system requires an understanding of smart devices basics to make sense [10]. In terms of interaction design, for smart devices, the interaction is mainly implemented by gestures on the screen. And the problem lies in that motor-impaired PD patients cannot accurately tap on a small icon on the screen or perform those interaction gestures well, like sliding or scrolling. Buttons of touch screens react too fast and this is a problem for the elderly [4]. Audio interaction is also not feasible as PD has strong effects in patients' language competence, which result in their lack of clarity and loudness and make it hard for ASR to recognize what they are saying [22]. What's more, spreading information across screens or forcing scrolling may be problematic due to working memory decline [2] and decline in ability to coordinate multiple tasks using attention switching [3]. Screens which present too many options or buttons may become confusing due to patients scanning the whole screen towards their goal rather than zeroing in, by visual cues, on what they are looking for [10]. Considering physical capabilities, smartphones usual keypad is not good for elderly and the physical buttons from mobile phones are too small. Design of the size should consider the trade-off between the screen size and handset size for patients to hold. As for the volume of audio interaction, when some instructions of a UI want to be given by voice, patients with audio impairment cannot receive the signal in some frequencies range [1]. Consider whether to listen carefully to the elderly, but also need to consider the impact of the size of the sound on vision [12].

To provide an optimal design to the intended audience, we designed and developed PD-Helper to satisfy the patients' need of communication, entertainment and requirement for medical care. With consideration of usability, accessibility and customization, application is developed based on scanning technique to provide single touch interaction over entire screen of smart devices. Personalization feature allows patients to adjust the font size, background color and scanning speed according to one's individual pace. 40 users with PD, participated in the evaluation process of the prototype. Preliminary results are promising and indicate a good level of acceptance. The contributions of this study are that 1) an easy and feasible solution that helps PD patients to enhance their interaction with smart devices 2) A single platform that provides access to four major daily life utilities to satisfy the fundamental needs of PD patients 3) To accommodate the variant conditions 
of PD Patients, they can choose controllable speed, and entire screen as touch keypad helps to improve the accuracy, single touch interaction smoothly fits to their physical limitations and helps to reduce physical fatigue. 4)This research also insights that by improving the accessibility and usability of interaction design of smart devices, they can be really helpful to provide a more productive, independent, social and fulfilling life to elder society.

\section{RELATED WORK}

Assistive technology is trying to assist the elderly society and motor impaired by providing a variety of applications to cope with interaction challenges in web search, text input as well as for health care. Keyboard is an essential modularity to interact with mobile phones. Researchers tried to optimize the QWERTY keyboard for a high efficiency by changing the layout [21], changing the key size, width or color [17,18] and different kinds of gestures such as swipe [19], shake [14] and other hand gestures [16] are used to promote typing efficiency. For web browsing, [11] developed a browser allowed patients to choose the links by presenting a series of brain responses within limited time. Web Accessibility Initiative provides bigger text with larger clicking areas and patients can order a special mouse to deal with the problem of shaking hands. For patients' Health care system, [7] did a survey to determine participants' perceptions of whether PROME would facilitate patientphysician communication. For multimedia, [23] created a BrainComputer-Interaction system based on electroencephalography (EEG) signals to meet the recreation need of people with severe disability.

Though the development of the IME seems highly improved the efficiency of typing for healthy people, but the number of keys and the complexity of gestures increase difficulty for patients whose hands are shaky to tap or follow the gestures that is why systems [14-21] are not suitable for PD patients.

The browsers which have been developed [9, 11, 23, 24] have a strong limitation due to precise use of mouse control movement, hence is not adaptable to PD patients. It is more important for doctors to get patients feedback to decide whether the medicine is suitable or not $[6,7]$. But the need to access medical services directly by expressing patient feeling is still need to pay attention.

Multimedia and recreation content access is really helpful to change the mind state of patients especially when they feel depressed. PD patients are incapable to use most of them [23]. All above mentioned problems highlight the need of our proposed PD-Helper system that can help PD patients to interact with smart device applications according to their physical capabilities. The details are given below in the design section.

\section{APPLICATION DESIGN}

To make our design in accordance with PD patients' needs and physical capabilities, we have considered following aspects: accessibility, simple design without intervention of wearable sensors and additional hardware and interaction technique should be feasible for PD patients; usability, the system is easy to learn and use; customization, users are allowed to make adjustment to the system settings according to their own controllability. We carefully considered and applied the three aspects in user interface design, interaction design and physical capabilities adaptation.

\section{A. User interface design}

- Main interface: PD-Helper provides four modules, namely website browsing, multimedia access, notebook and health care. As for the layout of the four modules and main interface, we adopted a neutral color scheme as background color and highlighting colors as icons' color, in order to ease the visual burden for PD patients to distinguish the flashing icons. The layout is concise and consistent in the whole system. Each icon and graphic representing modules or functions are easy to identify and are displayed with a larger size than that of the traditional mobile systems.

- IME interface: The layout is designed with 7 keys and one large size touch pad to make the interaction easier and efficient for motor impaired patients. First the initial alphabet (consonant) of pinyin is displayed. After that the system will show the possible pinyin combination. By selecting desiring combination, system will display the Chinese characters. The desired choices in each step are displayed within 7 keys.

\section{B. Interaction design: Single touch and menu selection}

PD-Helper provides a single touch interaction and whole screen as input area for users. Considering the trembling or rigidity symptoms, single touch allows users to touch the screen for a very short time to realize input and the application sets a large touch pad for them to touch and decrease the probability of error. Users do not click over individual keys and alphabets to select, they can tap over the entire area to make a selection. Menu selection is realized using scanning technique rather than touching on the small area of an icon. When the target items start to flash in turn, users can touch anywhere on the screen to select them.

\section{Input technique: IME and shortcut menu}

Consonant Character Input Method [25] is used in the IME application. To control the interaction via single touch, scanning ambiguous keyboard concept is used, because this is an efficient way to implement the system with reduced size and control the output via single key [23]. Sequence of typing one word is as follows. First look up for the initial alphabet (consonant) of pinyin. After that system will show the possible pinyin combination. By selecting desiring combination, system will display the Chinese characters. A small offline database connects the IME application to retrieve the Chinese characters efficiently. To maintain the efficiency during whole typing process, an online database is connected. So that if user does not find desired character, he just need to select the " $x$ ", system will type pinyin directly in the textbox. After user finished one sentence, system will replace pinyin with corresponding Chinese character.

\section{Physical capabilities adaptation}

A design should meet the patients' physical capabilities to make it adaptable at user level. Fig. 1 shows the difference between device interaction and user capabilities to interact with 
them. As interaction techniques based on precise target selection are out of questions for the patients, so it is really challenge for them to cope with these devices.

PD-Helper uses scanning technique to make selection, Icons flash in turn and users can touch on the entire screen to realize their input. Single tap input allows users to input with least physical effort. In this way, these applications accessible to more people with physical disabilities. Personalization settings are provided to change the scanning speed $(4 s, 3 s, 2 s)$, and color theme to facilitate the diverse conditions of PD. The web user controls with scanning technique and video easy control help patients to access the applications.

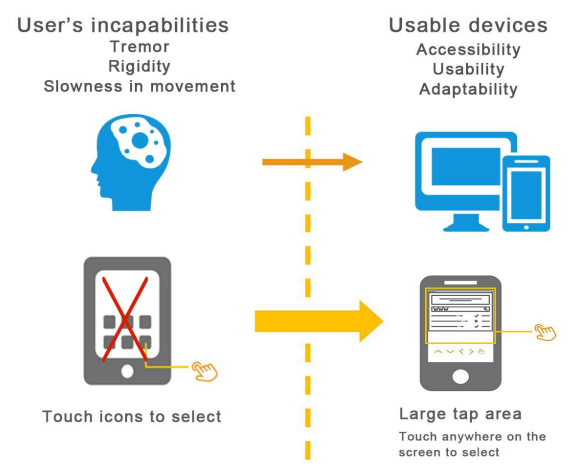

Figure 1. Physical capabilities adaptation

\section{APPLICATION IMPLEMENTATION}

To implement the proposed design, we developed PD-Helper for Android platform. PD-Helper is composite of four modules, namely web browsing, multimedia, notebook and healthcare and provide patients with social connectivity, medical care and entertainment. The detailed implementation of each module in the application is listed below:

\section{A. Main menu}

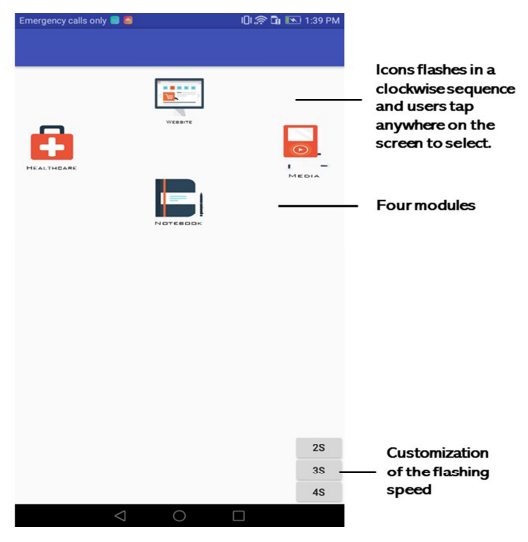

Figure 2. Main menu of PD-Helper

Main page displays four modules and their icons flash clockwise from website to multimedia, then notebook and the lastly healthcare (see Fig.2). Lower most right settings help to change the scan interval speed of the entire application. The selection in PD-Helper can be made by single tap anywhere on the entire screen.

\section{B. Web browsing}

This module allows patients to browse the internet. When user opens browser, internet browser page divides into 2 parts: page control area and web control area (see Fig.3). Web page control area consists of web controls that start to scan in turn and helps to increase and decrease the font, refresh the current page, open new page and return to main menu by single tapping over the screen.

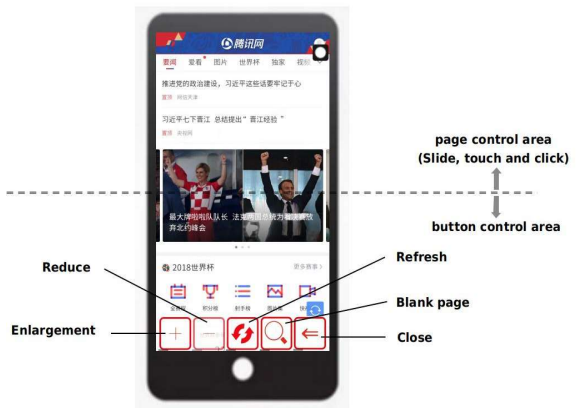

Figure 3. Web-Browsing PD-Helper

\section{Multimedia}

Video source: Multimedia Access provides a list of videos for users to watch, including some classic movies and rehabilitation treatment videos (see Fig.4)

Easy control: users touch the screen to select the video, and it plays automatically. Users can pause and play by single tapping for a short time, and then tap it again to continue to play. Touching the screen for a bit long time can exit the video and return to the video list. This mechanism is easy for PD patients to control videos.

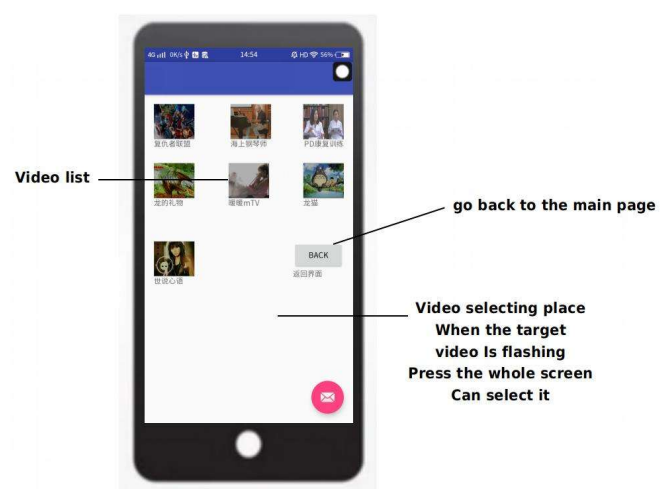

Figure 4. Multimedia module of PD-Helper 


\section{Notebook}

This module provides an IME application that designed specifically for PD patients to type Chinese character in the text input area via single touch. Notebook application helps to type text, save notes, record notes and view the previous records. Users can also browse some relevant articles by typing keyword via "search" function. It provides many motivational cards with pictures and some motivational words to encourage users to change their mood. The content of the cards will be changed with tapping on the screen. Patients often have trouble in sleeping well due to physical therapy and psychological stress. The subsection "Sleep" provides many hypnotic music to improve PD patients' sleep quality (see Fig.5).

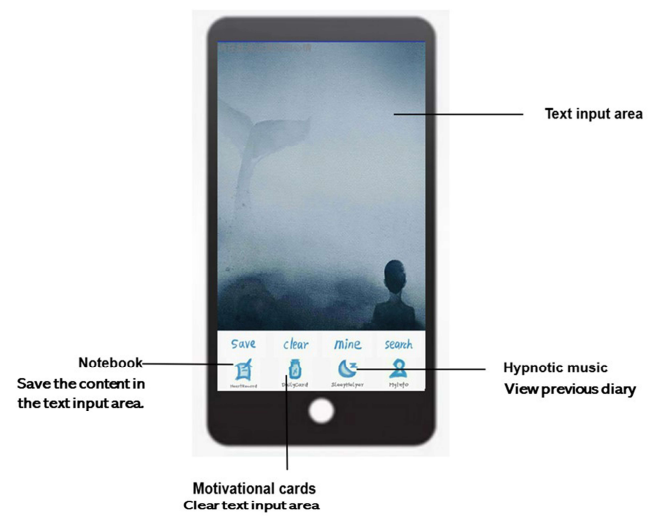

Figure 5. Notebook module of PD-Helper

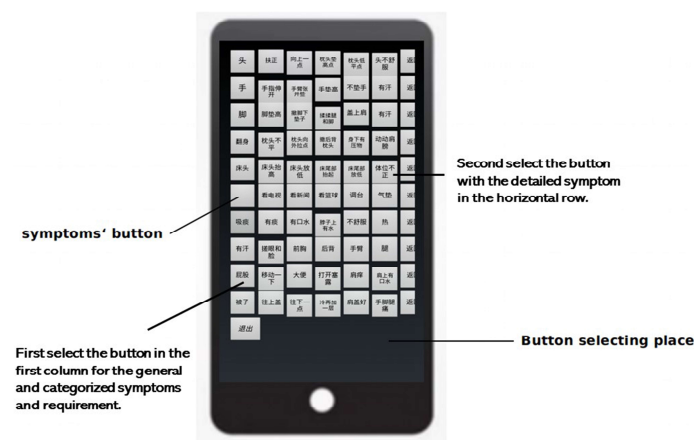

Figure 6. Health care connectivity

\section{E. Healthcare}

PD patients may suffer from speech impairment and are unable to express their need. This module provides common possible symptoms and needs for patients to express their feelings to the medical care staff automatically via the message system (see Fig. 6). The system helps to select the category of symptoms from the column via scanning technique and then pick the detailed symptoms in the corresponding row via single tap over the entire screen. The message will be sent to the contact number of care taker staff.

\section{USER STUDIES}

We conducted user studies to test the usability and to determine the effects of PD-Helper with PD patients. 40 recruited patients ( 18 males, 22 females) with age ranging from 43 to 89 (mean=69; $\mathrm{SD}=11.03$ ) have been divided into two group according to their PD symptoms, namely tremor (T) and rigidity(R). Neurologist helped us to make selection of patients based on information from hospital records and nursing staff. Patient inclusion criteria includes 1) Parkinson's disease symptoms (Rigidity, Tremor), 2) off state. Neurologist helped to motivate the patients to take part in the user study voluntarily. Neurologists helped to collect UDPRS data of participants. We divided the patient's disease degree of severity into 3 levels only (using UDPRD data). All the patients signed informed patient consent form willingly and voluntarily participated to test the system.

To test the adaptability and accessibility of PD-helper with patients an experiment with 30 min time span is conducted. Keeping in view patient physical condition, it was tiring for a patient to test all the modules of PD helper. To test each module, we have divided patients into groups based on their capability to test the different modules. 22 Patients who were familiar with pinyin helped to test the notebook/IME module. The 18 patients with no or little knowledge of smart devices helped to test the web browsing and multimedia module. During the test sessions, participants had to perform the following tasks with three different scanning speeds.

Task 1: play the third video from the multimedia module, and check the play, pause and close functions.

Task 2: Patient has to open SOHU website and used the all available web user controls to increase, decrease font, refresh the page, open new page, and back to main menu.

Task3: Type a random sentence (selected from the old Chinese poem and daily life sentences) with the note book module (see Table III).

Test was conducted on Tablet $(7.5$ inches screen, android OS). Patients performed the test while holding the tablet in assistant hand, in their own hand and over the table as well.

\section{A. Results and data of Task 1}

18 participants took part to complete the video test. During test session, patient has to play a video, then use play, pause and close features of the application that are specifically designed to assist the diverse conditions of PD. The aim of the test is to measure the accuracy rate of users' operation with different speed interval on video task. Before the test, we estimated the time required to complete the task without error and the estimated time (ET) for 2-4s speed interval is respectively $71.6 \mathrm{~s}$, $73.6 \mathrm{~s}$ and $75.2 \mathrm{~s}$. We then measured the actual time (AT) participants took to complete the task in the test. Accuracy rate calculated in the following way:

$$
\mathrm{R}(\text { Accuracy rate })=1-100 \% *(\mathrm{AT}-\mathrm{ET}) / \mathrm{AT}
$$

TABLE I. ACCURACY AND ERROR RATE OF TASK 1

\begin{tabular}{|l|c|c|c|c|}
\hline \multirow{2}{*}{$\begin{array}{c}\text { Speed } \\
\text { Interval }\end{array}$} & \multicolumn{2}{|c|}{ Accuracy Rate } & \multicolumn{2}{c|}{ Error Rate } \\
\cline { 2 - 5 } & $\boldsymbol{T}$ & $\boldsymbol{R}$ & $\boldsymbol{T}$ & $\boldsymbol{R}$ \\
\hline $4 \mathrm{~s}$ & 95.4 & 91.9 & 4.6 & 8.1 \\
\hline $3 \mathrm{~s}$ & 88.0 & 89.9 & 12.0 & 10.1 \\
\hline
\end{tabular}




\begin{tabular}{|l|c|c|c|c|}
\hline \multirow{2}{*}{$\begin{array}{c}\text { Speed } \\
\text { Interval }\end{array}$} & \multicolumn{2}{|c|}{ Accuracy Rate } & \multicolumn{2}{c|}{ Error Rate } \\
\cline { 2 - 5 } & $\boldsymbol{T}$ & $\boldsymbol{R}$ & $\boldsymbol{T}$ & $\boldsymbol{R}$ \\
\hline $2 \mathrm{~s}$ & 78.7 & 80.5 & 11.3 & 19.5 \\
\hline
\end{tabular}

\section{B. Results and data of Task 2}

18 participants took test in web browsing module and all of them have completed the test. The test aims at testing the usability of web browsing mechanism and measuring the accuracy rate of users' operation with different speed interval. Before the test, we estimated the time required to complete the task without error and the estimated time (ET) for 2-4s speed interval is respectively $30 \mathrm{~s}, 40 \mathrm{~s}$ and $50 \mathrm{~s}$. We then measured the actual time (AT) participants took to complete the task in the test. Accuracy rate calculated by equation (1).

TABLE II. ACCURACY AND ERROR RATE OF TASK 2

\begin{tabular}{|l|c|c|c|c|}
\hline \multirow{2}{*}{$\begin{array}{c}\text { Speed } \\
\text { Interval }\end{array}$} & \multicolumn{2}{|c|}{ Accuracy Rate } & \multicolumn{2}{c|}{ Error Rate } \\
\cline { 2 - 5 } & $\boldsymbol{T}$ & $\boldsymbol{R}$ & $\boldsymbol{T}$ & $\boldsymbol{R}$ \\
\hline $4 \mathrm{~s}$ & 92.2 & 98.7 & 7.8 & 1.3 \\
\hline $3 \mathrm{~s}$ & 85.3 & 90.4 & 14.7 & 9.6 \\
\hline $2 \mathrm{~s}$ & 77.6 & 79.9 & 22.4 & 20.1 \\
\hline
\end{tabular}

\section{Results and data of Task 3}

22 Participants overall have been divided according to their degree of severity to test the correlation between degree of severity, different speed interval $(1 \mathrm{~s}, 2 \mathrm{~s}, 3 \mathrm{~s})$ and typing speed.

Patient used Table III sentences to complete the task 3. By keeping in view patients' physical condition short random sentences are preferably selected to avoid from tiredness and physical fatigue. In 1s speed interval test, the numbers of people with degree of severity from 1 to 3 are respectively $4,13,5$. In 2 s speed interval test, there are 19 people with 2 degrees of severity and 3 people with 3 degrees of severity. In 3 s speed interval test, 10 people are with 2 degrees of severity and 12 people are with 3 degrees of severity.

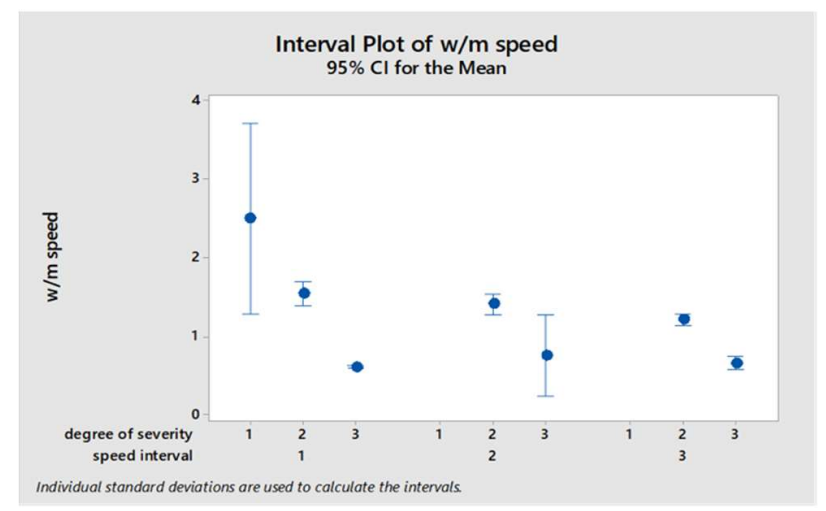

Figure 7. Interval Plot of $\mathrm{w} / \mathrm{m}$ speed

In terms of speed interval in relation to typing speed (Fig. 7), people have an approximately average typing speed of 2 words/minute with $1 \mathrm{~s}$ interval, 1.1 words/minute with $2 \mathrm{~s}$ interval, 0.65 word/minute with $3 \mathrm{~s}$ interval. There's negative linear relationship between degree of severity and speed of typing. People have an approximately average typing speed of 2.3 words/minute with 1 degree of severity, 1.5 words/minute with 2-degree of severity, 0.7 word/minute with 3-degree of severity.

TABLE III. TEST SENETNCES FOR TASK 3

\begin{tabular}{|l|l|l|}
\hline \multicolumn{1}{|c|}{ Sentence } & \multicolumn{1}{c|}{ Pinyin } & \multicolumn{1}{c|}{ Meaning } \\
\hline 谢谢 & xie xie & Thanks \\
\hline 没问题 & Mei wen ti & No problem \\
\hline 我在北京 & Wo zai bei jing & I'm in Beijing. \\
\hline $\begin{array}{l}\text { 妈 妈 有 三 } \\
\text { 个孩子 }\end{array}$ & $\begin{array}{l}\text { Ma ma you san ge hai } \\
\text { zi }\end{array}$ & Mom has three children \\
\hline $\begin{array}{l}\text { 欢迎使用这个系 } \\
\text { 统 }\end{array}$ & $\begin{array}{l}\text { Huan ying shi yong zhe } \\
\text { ge xi tong. }\end{array}$ & $\begin{array}{l}\text { Welcome to use this } \\
\text { system }\end{array}$ \\
\hline
\end{tabular}

\section{DISCUSSION}

From above data results section, it is proved that single touch PD-Helper is effective for patients to access the four major daily life utilities with more ease and accuracy via smart devices. The reason was that touch interaction technique was based on user experience and it was easy for the patients to adapt the one touch interaction technique as they were already familiar with it rather than learning novice and complex gestures. All patients used the three flash speeds to complete the test irrespective of their severity. During task 1 tremor patients showed more accuracy $95 \%$ with $4 \mathrm{~s}$ as compared to rigidity patients $91 \%$. During task 2 the lowest rate for accuracy was $77 \%$ and $79 \%$ of tremor and rigidity respectively. That shows really good acceptance especially in case of PD patients. It showed that patients adapt only those features that are in accordance with their capabilities. User's questionnaire feedback shows that IME is easy to use due to simple consonant vowel structure and personalized features make it suitable for diverse severity patients. The proposed system is best combination of accessibility, accuracy and usability.

Accessibility: Different scanning speed level can be adjustable according to patient physical condition, so patients with diverse conditions can access PD-Helper without any discomfort

Accuracy: large size icons, font size and color theme provide more clarity and visibility to the patients. A fatigue of exact target selection is also eliminated. To make selection of target they can touch anywhere of the screen. It helped to increase the accuracy for patients.

Usability: single tap selection along with large touch area reduce their motoric interaction and hence physical fatigue for them.

Fig.8 shows the user study performed with patients. This user study has made major relevant contributions in minimizing the motoric interaction for PD patients to access the smart devices. The idea of PD-Helper via single tap interaction over smart 
devices is introduced for the first time for PD patients that is why the comparative study was not possible. All users appreciate the idea and practical worth of this system

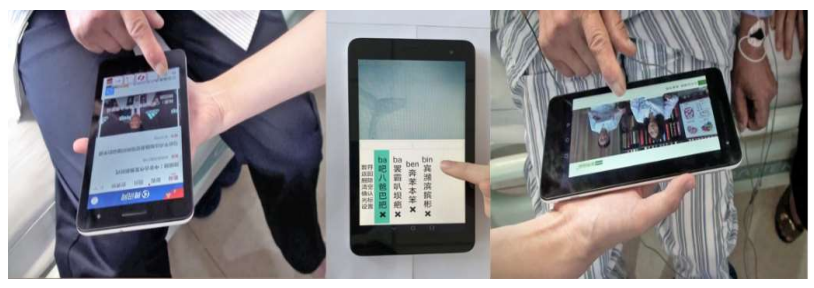

Figure 8. User Study with PD-Helper Patients in Xuan Wu Hospital

\section{CONCLUSION}

Parkinson's disease (PD) Patients with diverse physical conditions exhibit challenges in interacting with smart devices due to motor impairment. To enhance their interaction, and accessibility with smart devices, PD-Helper is designed and developed with single touch interaction as well as a large touch pad area to improve tactile experience for intended audience. Scanning technique is used to control input via single tap to minimize the physical fatigue for patients. By virtue of personalization features 40 participants (PD patients) were able to access the web, multimedia content and input Chinese text with more accuracy. The IME application, Web user controls, video easy controls, and connectivity with health care staff helps PD patient to improve their social connectivity via smart devices. Further studies are in progress to attest the PD helper potential, such as improving the quality of life of people with other Neurological disorders. Current limitation of the system is that it is developed for Chinese language. As a future work, a multilingual PD-Helper solution will be offered to support the patients around the globe.

\section{ACKNOWLEDGEMENT}

The project is supported by the Project: 61672017 of National Science Foundation of China.

\section{REFERENCES}

[1] Carmien S., Manzanares A.G. (2014) Elders Using Smartphones - A Set of Research Based Heuristic Guidelines for Designers. In: Stephanidis C., Antona M. (eds) Universal Access in Human-Computer Interaction. Universal Access to Information and Knowledge. UAHCI 2014. Lecture Notes in Computer Science, vol 8514. Springer, Cham

[2] Fisk, A.D., Rogers, W. A., Charness, N., Czaja, S. J., \& Sharit, J. , Designing for Older Adults: Principles and Creative Human Factors Approaches2004: CRC Press J Taylor\&.Francis Croup

[3] Jacko JA, Barreto AB, Marrnet GJ, Chu JYM, Bautsch HS, Scott IU, Rosa RH (2000) Low vision: the role of visual acuity in the efficiency of cursor movement. In: Proceedings of ASSETS'00, Arlington, VA, November 2000. ACM Press, pp 1-8

[4] Strengers, J., Smartphone interface design requirements for seniors, in Information Studies2012, University of Amsterdam: Amsterdam.

[5] Kurniawan, S. and P. Zaphiris, Research-derived web design guidelines for older people, in Proceedings of the 7th international ACM SIGACCESS conference on Computers and accessibility2005, ACM: Baltimore, MD, USA. p. 129-135.
[6] Krpic, Andrej et al. "Telerehabilitation: remote multimedia-supported assistance and mobile monitoring of balance training outcomes can facilitate the clinical staff's effort." International journal of rehabilitation research. Internationale Zeitschrift fur Rehabilitationsforschung. Revue internationale de recherches de readaptation 362 (2013): 162-71.

[7] Hong, Song Hee et al. "Health Care Applicability of a Patient-Centric Web Portal for Patients' Medication Experience." Journal of medical Internet research (2016).

[8] Francisco Nunes $\bullet$ Paula Alexandra Silva $\bullet$ Joa o Cevada $・$ Ana Correia Barros • Lu1 's Teixeira, User interface design guidelines for smartphone applications for people with Parkinson's disease, Univ Access Inf Soc (2016) 15:659-679 DOI 10.1007/s10209-015-0440-1

[9] Hanson, Vicki L. and Susan Crayne. "Personalization of Web browsing: adaptations to meet the needs of older adults." Universal Access in the Information Society 4 (2005): 46-58.

[10] Chisnell, D., Redish, J. Designing web sites for older adults: a review of recent research. AARP.org/olderwiserwired, 2004.

[11] Bensch, Michael et al. "Nessi: An EEG-Controlled Web Browser for Severely Paralyzed Patients." Computational Intelligence and Neuroscience 2007 (2007): 297 - 298.

[12] Minggang Yang and He Huang, Research on Interaction Design of Intelligent Mobile Phone for the Elderly Based on the User Experience

[13] Rodrigues, Élvio, Micael Carreira, and Daniel Gonçalves. "Developing a multimodal interface for the elderly." Procedia computer science 27 (2014): 359-368

[14] Mark Dunlop, Andreas Komninos, Emma Nicol, and Iain Hamiliton. 2014. Shake ' $n$ ' Tap: a gesture enhanced keyboard for older adults. In Proceedings of the 16th international conference on Human-computer interaction with mobile devices \& services (MobileHCI '14). ACM, New York, NY, USA, 525-530.

[15] Hutchison, Elizabeth A et al. "Diversification of a protein kinase cascade: IME-2 is involved in nonself recognition and programmed cell death in Neurospora crassa” Genetics vol. 192,2 (2012): 467-82.

[16] Yin, Ying et al. "Making touchscreen keyboards adaptive to keys, hand postures, and individuals: a hierarchical spatial backoff model approach." CHI (2013).

[17] Hsiao HC, Wu FG, Chen CH. Design and evaluation of small, linear QWERTY keyboards. Appl Ergon. 2014 May;45(3):655-62. doi: 10.1016/j.apergo.2013.09.001. Epub 2013 Sep 26. PubMed PMID: 24075287.

[18] Antti Oulasvirta, Anna Reichel, Wenbin Li, Yan Zhang, Myroslav Bachynskyi, Keith Vertanen, and Per Ola Kristensson. 2013. Improving two-thumb text entry on touchscreen devices. In Proceedings of the SIGCHI Conference on Human Factors in Computing Systems (CHI '13). ACM, New York, NY, USA, 2765-2774.

[19] Sakkos, Panos et al. “Anima: Adaptive Personalized Software Keyboard." CoRR abs/1501.05696 (2015): n. pag.

[20] Bi, Xiaojun et al. "Multilingual Touchscreen Keyboard Design and Optimization.” Human-Computer Interaction 27 (2012): 352-382.

[21] Bi, Xiaojun, Barton A. Smith and Shumin Zhai. "Quasi-qwerty soft keyboard optimization.” CHI (2010)

[22] Ondrej Polacek1 • Adam J. Sporka1 - Pavel Slavik1, "Text input for motor-impaired people", Univ Access Inf Soc (2017) 16:51-72, DOI 10.1007/s10209-015-0433-0

[23] Jabeen, Farzana et al. "Mind interactive multimedia system for disabled people." 2017 10th International Congress on Image and Signal Processing, BioMedical Engineering and Informatics (CISP-BMEI) (2017): 1-6.

[24] Poulson, David and Colette Nicolle. "Making the Internet accessible for people with cognitive and communication Impairments." Universal Access in the Information Society 3 (2003): 48-56.

[25] Jabeen, Farzana, Tao, Linmi, Wang, Xinyue, et al. C-SAK: Chinese Scanning Ambiguous Keyboard for Parkinson's Disease Patients. In : 2018 IEEE 16th Intl Conf on Dependable, Autonomic and Secure Computing, 16th Intl Conf on Pervasive Intelligence and Computing, 4th Intl Conf on Big Data Intelligence and Computing and Cyber Science and Technology Congress (DASC/PiCom/DataCom/CyberSciTech). IEEE, 2018. p. 792-799. 